\title{
Synchronous Primary Carcinoma of Cervix and Endometrium
}

\author{
Divya Madhala', Gouthaman Shanmugasundaram² ${ }^{2}$, Sai Shalini Chinnathambi Narayanan ${ }^{1}$ and Jaya Vijayaraghavan $^{3}$ \\ ${ }^{1}$ Department of Pathology, Sri Ramachandra Medical College and Research Institute, Chennai, India \\ ${ }^{2}$ Department of Surgical Oncology, Sri Ramachandra Medical College and Research Institute, Chennai, India \\ ${ }^{3}$ Department of Obstetrics and Gynecology, Sri Ramachandra Medical College and Research Institute, Chennai, India
}

\begin{abstract}
Introduction: Synchronous primary tumours in the female genital tract are rare. Even rarer are the synchronous tumours involving the cervix and endometrium . We describe a rare case of cervical squamous cell carcinoma and endometrial endometrioid adenocarcinoma in a 62-yearold woman .

Case Report: A 62-year-old female presented with post menopausal bleeding for 4 months duration. Per vaginal examination showed a cervical growth. Magnetic resonance imaging revealed a mass in the cervical region and extension into the body and fundus of the uterine cavity. Radical hysterectomy(Type III hysterectomy) was done. The pathology revealed synchronous occurrence of primary neoplasms of squamous cell carcinoma of the cervix and adenocarcinoma of the endometrium.
\end{abstract}

Conclusion: Synchronous malignancies of endometrium and cervix are rare. Surgical treatment is radical surgery- Type 3 hysterectomy with bilateral pelvic lymph node dissection. The knowledge of occurrence of synchronous primaries is needed to plan appropriate adjuvant treatment.

Keywords: Cervix, Endometrium, Cancer, Pathology

\section{Introduction}

Synchronous tumours are two or more histologically distinct malignancies detected simultaneously. Synchronous primary malignancies of the female genital tract are very rare. The most common combination being ovarian and endometrial primary neoplasms ${ }^{[1]}$.Very few cases of synchronous malignant tumours of endometrium and cervix have been reported in the world literature ${ }^{[2]}$. The prognosis of these patients depends on the stage of presentation of these malignant tumours. We present a very rare case of synchronous tumour of endometrial and cervical cancer in a 62 year old female patient.

\section{Case Report}

A 62 year old lady presented with complaints of intermittent post menopausal bleeding for 4 months. Patient also had white discharge per vaginum. She is a known diabetic and hypertensive. Pap smear examination revealed malignant squamous cells following which she was referred to our center for further management.

Per abdomen examination was within normal limits. Per vaginal examination showed a ulcero-proliferative growth obstructing the cervical os from which cervical biopsy was taken. The histopathology of the cervical biopsy showed features of poorly differentiated squamous cell carcinoma.

Magnetic resonance imaging showed a bulky cervix with partially circumferential growth predominantly involving the anterior and right lateral walls. Tumour extended into body and fundus of uterus with $>50 \%$ of myometrial thickness involvement(Figure 1). No serosal involvemt was present. The parametrium was free and pelvic side walls appeared normal. Few bilateral internal iliac nodes were noted. In view of disease confined to the cervix with no parametrial invasion, type 3 hysterectomy with bilateral salphingo - oopherectomy and bilateral pelvic lymph node dissection was planned.

Examination under anesthesia was done which showed no parametrial involvement. Maylards incision was made. Exploration of the abdomen was done. There was no significant pelvic or para aortic nodes enlargement. Uterine artery was ligated at the origin. Ureter dissected till bladder base from pelvic brim. Parametrium was divided close to the pelvic side walls. Utero-sacral ligaments were divided close to rectal wall. Upper one third of vagina removed en-bloc with the specimen. Bilateral pelvic lymph node dissection was done.

We received total abdominal hysterectomy with bilateral salphingo-oopherectomy specimen along with bilateral pelvic lymph nodes. Uterus with cervix and bilateral tubes and ovaries weighed $30 \mathrm{~g}$. Uterus with cervix measures $8.5 \times 5 \times 2 \mathrm{~cm}$. Parametrial length was $6 \mathrm{~cm}$ on each side, cervix external surface was unremarkable. Cut surface showed a ill defined, infiltrative, grey white firm lesion measuring $2 \times 2 \mathrm{~cm}$ and depth of $1.4 \mathrm{~cm}$. Tumour is seen 
involving the endo-cervical region. Serosal surface is free of tumour. Distance from the vaginal cuff was $0.7 \mathrm{~cm}$. Endometrium showed a irregular grey white to grey brown proliferative mass measuring $5.5 \times 2.5 \times 0.7 \mathrm{~cm}$ (Figure 2), and myometrium measures $1 \mathrm{~cm}$. Both ovaries and tubes were unremarkable.

Microscopically, cervix showed a FIGO grade 3 poorly differentiated non keratinizing squamous cell carcinoma of cervix with adjacent areas showing cervical dysplasia(Figure 3). Few areas of necrosis was also identified(Figure 4). Endometrium showed a FIGO grade 1 well differentiated, Endometrioid adenocarcinoma not

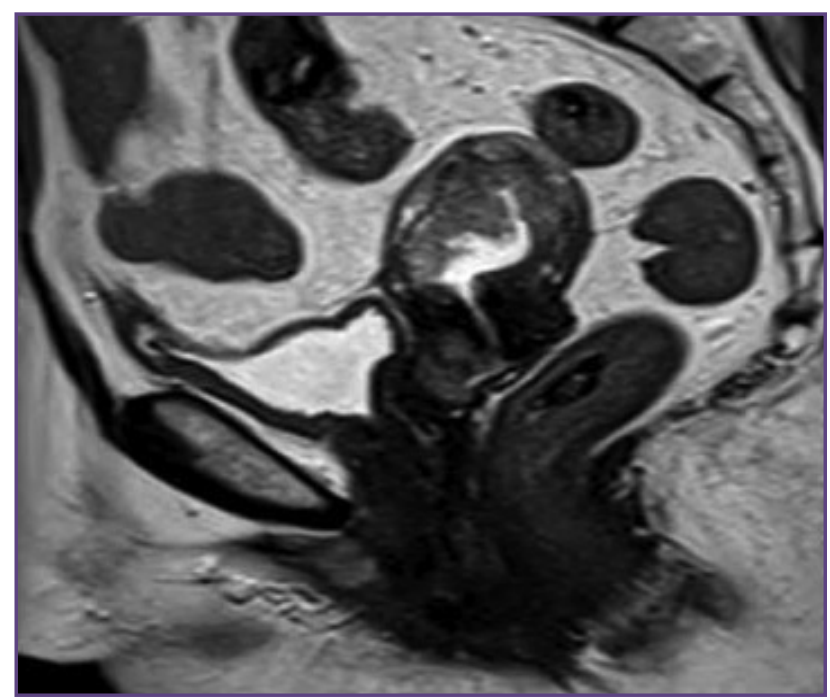

Fig. 1: MRI of cervix and endometrium.

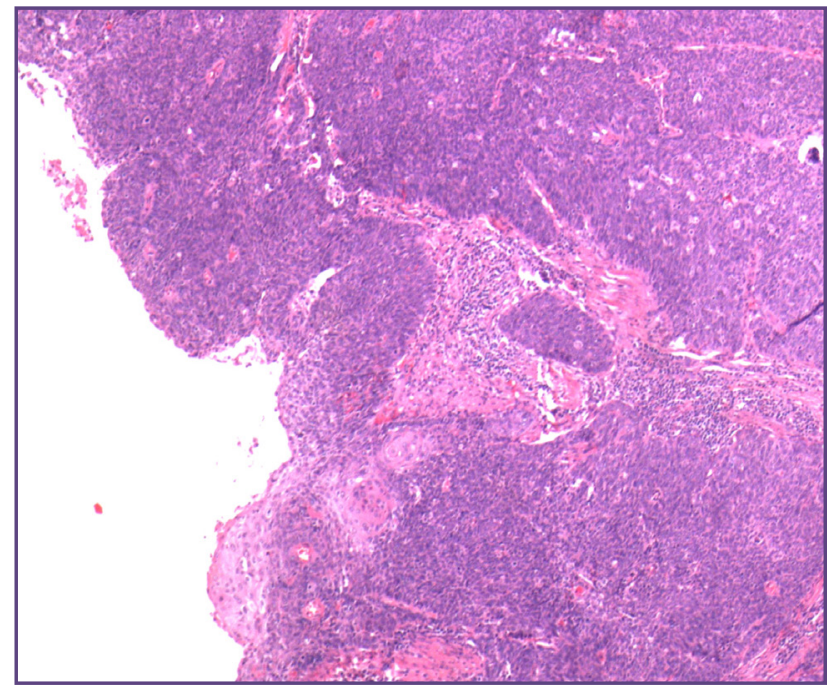

Fig. 3: Section shows Cervical carcinoma with dysplasia (H\&E, 20x). otherwise characterized( Figure 5, Figure 6). Margins uninvolved. The bilateral ovaries, tubes, omentum, parametrium and vaginal surgical margins were all free. Surgical vaginal cuff was free of tumor. Also, there was no lower vaginal segment involvement of the endometrial cancer. Bilateral pelvic lymph nodes were free of tumour (27 lymph nodes were examined).

Based on pathological examination, a diagnosis of FIGO stage IB1 cervical malignancy and FIGO stage IB endometrial malignancy was made. Following surgery patient was started on adjuvant external beam radiation therapy.

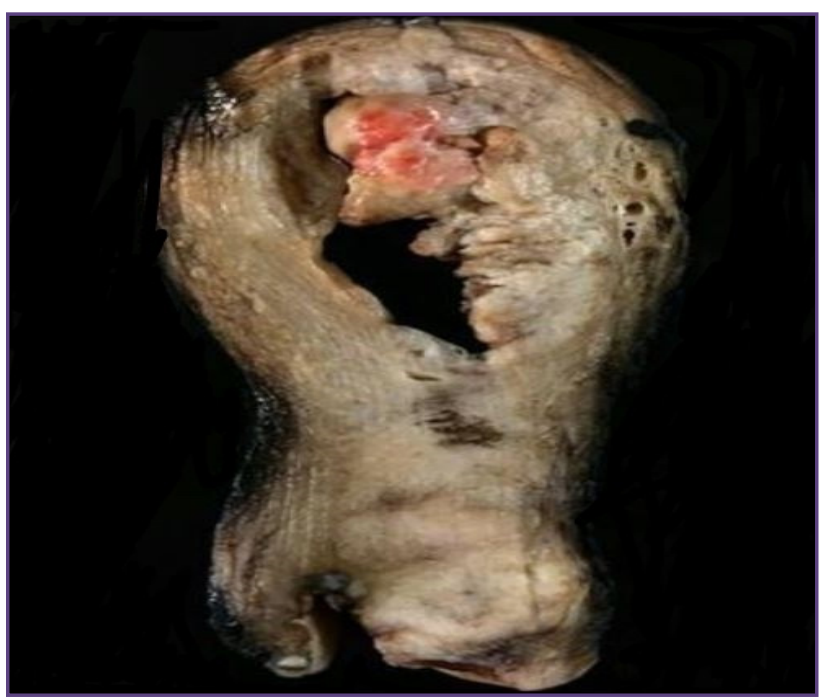

Fig. 2: Cervix showing a ill defined, infiltrative, grey white firm lesion and endometrium with irregular grey white to grey brown proliferative mass.

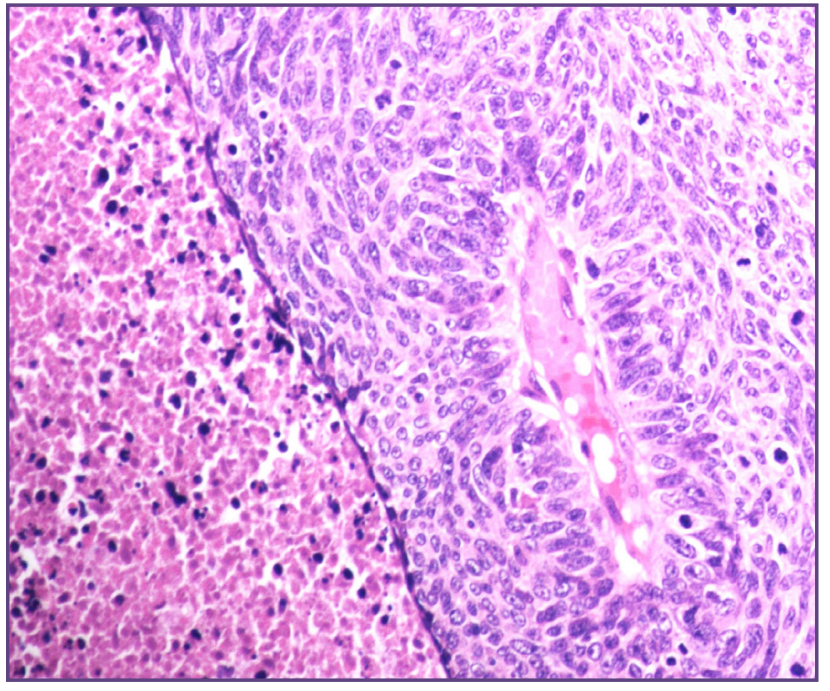

Fig. 4: Section showing Cervical carcinoma with atypical mitosis and areas of necrosis (H\&E, 20x). 


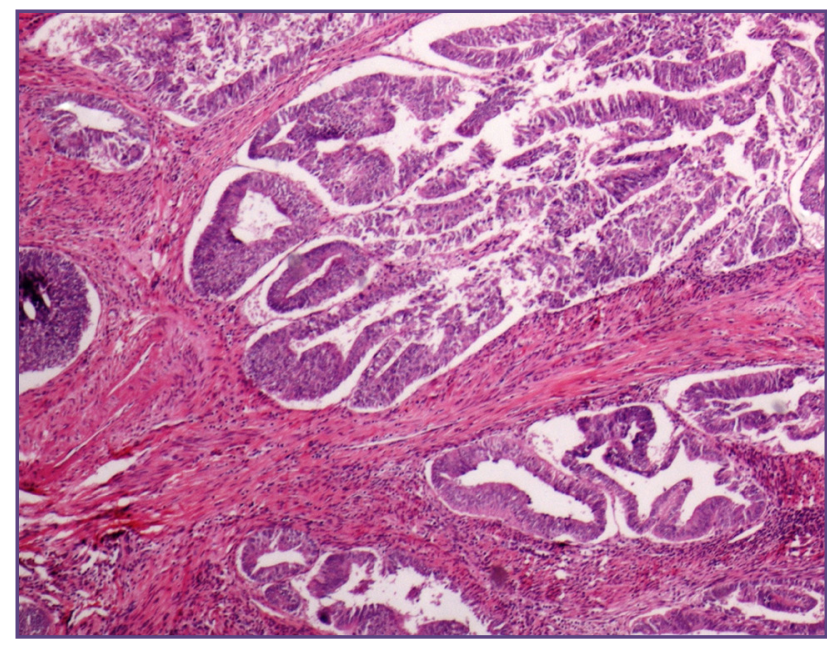

Fig. 5: Section showing Endometrial endometrioid adenocarcinoma (H\&E, 4x).

\section{Discussion}

Multiple primary tumours can be synchronous or metachronous. Synchronous tumours are two or more histologically distinct simultaneously detected malignancies. The incidence of $1 \%-6 \%$ shows the rarity of these tumours. Out of this the most common synchronous tumour is that of ovarian and endometrium.

The etiopathogenesis of these synchronous tumours is very rarely understood. It has been proposed that, embryologically similar tissues when simultaneously subjected to either hormones or carcinogens may develop synchronous neoplasms in genetically susceptible individuals. The concept of field cancerization ${ }^{[1,3]}$ which was earlier applied for tumours arising from head and neck region has being applied to the synchronous tumours of female genital tract. Here the repeated exposure of the mucosa of the female genital tract to multiple risk factors leads to the development of multicentric disease . It is suggested that cancers developing in different sites originate from histologically similar epithelium. This "secondary mullerian system" concept attempts to explain the etiology of synchronous primaries in female genital tract. The patients are usually post-menopausal with the median age of presentation being 50-60 years. Most patients present with post menopausal bleeding and vaginal discharge.

Pathologically Synchronous primary endometrial and cervical cancers may have similar or different histologic appearance. The distinction between synchronous primary and metastatic cancers is relatively easy, when they have different histologic types. However, the distinction is relatively difficult, when they have the same histologic type. Most common histopathology seen in endometrium

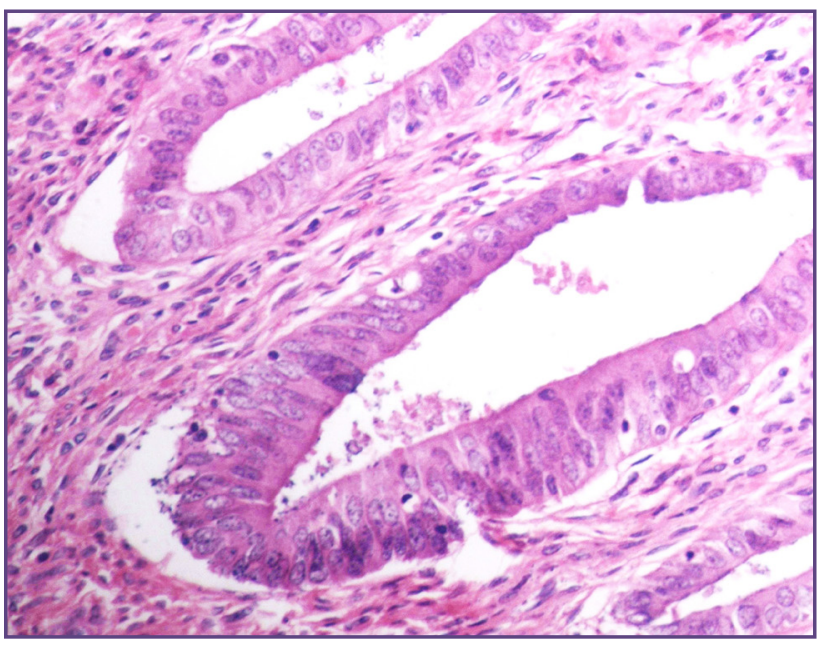

Fig. 6: Section showing endometrial endometrioid adenocarcinoma (H\&E, 20x).

malignancy is endometrioid adenocarcinoma and in cervix it is squamous cell carcinoma.

The surgical management of these patients include radical hysterectomy(Type III hysterectomy) with bilateral pelvic lymph node dissection in view of cervical involvement. Appropriate surgical staging provides diagnostic, prognostic and therapeutic benefits for women with synchronous primary malignancies. It facilitates appropriate adjuvant treatment as well. In the literature the cases of cervical and endometrial neoplasm occurring at the same time have rarely been reported.

A single case study of synchronous cervical and endometrial neoplasm was reported by Cheng-kuo lin et $\mathrm{al}^{[2]}$ from national defence center, Taiwan. They suggested that the second primary cancer in patients having endometrial cancer may offer an opportunity for early detection. In our study, patient was diagnosed to have cervical malignancy and subsequently diagnosed to have carcinoma of endometrium in uterine gross specimen after radical hysterectomy.

In a study by Seo-yun tong et $\mathrm{al}^{[4]}, 20$ patients were diagnosed with synchronous tumours , 13 patients had synchronous tumours in the female genital tract and 7 with one primary in the female genital tract and 1 primary in extragenital. The most common was endometrium and ovarian malignancy. Three patients showed primary malignancies of the endometrium and the cervix. All three were associated with adenocarcinoma of the endometrium and two had squamous cell carcinoma of cervix and one had neuroendocrine tumour of cervix. This study proves that synchronous malignancy of endometrium and cervix as seen in our case is very rare. 
A Turkish study by Sultan eser et $\mathrm{al}^{[5]}$ was carried out to determine the frequency of occurrence of synchronous female genital tumours from 1993 to 2005 . A total of 4185 cases were identified, off which 55 were synchronous tumours. 43 tumours were synchronous tumours of endometrium and ovary whereas just 4 cases of synchronous tumours of endometrium and cervix was present indicating that this combination is very rare. In their study they observed that Endometrioid adenocarcinoma was the most frequent component in a synchronous tumour. Our case also had endometrioid adenocarcinoma.

In one study by Axelrod jh et $\mathrm{al}^{[6]}$ in 1984 reviewed 78 synchronous or metachronous tumours from 2362 patients. Synchronus tumour pairs of cervix was found in combination with ovary,uterus and kidneys. Also synchronous pairs were found in combinations of endometrium and ovary,endometrium and rectosigmoid, ovary and breast. In this study the synchronous tumours of cervix and endometrium as seen in our study as well was rare when compared to other synchronous tumours.

A case of Cervical signet-ring cell carcinoma presenting as a synchronous primary carcinoma with uterine adenocarcinoma was reported by Lower WJ et al ${ }^{[7]}$ in 2009. They found that cervical signet cell carcinoma is very rare with only 11 cases reported in literature and that occurring in combination with uterine neoplasm is even more rarer. But our case was squamous cell carcinoma of cervix along with endometrial endometrial adenocarcinoma.

In one study by Derya et al ${ }^{[8]}$, where triple malignancies of cervical,ovary and endometrial well differentiated endometrioid type carcinoma was reported. The patient underwent radical hysterectomy, bilateral salphingooopherectomy, retroperitoneal lymph node dissection and peritoneal sampling. Post operatively patient received 6 cycles of chemotherapy with cisplatin and paclitaxel. In our study adjuvant external beam radiation was given as a treatment for both FIGO stage IB carcinoma cervix and FIGO stage IB endometrial carcinoma. Chemotherapy was not given in view of early stage of the malignancies.

The prognosis of patients with synchronous tumours is more favourable when compared to metastatic lesions of individual tumours. Also the stage at which the patient presents to the health center also determines the outcome.
Another important factor is the lymph node status of these patients. It defines accurately the extent of disease and determines the prognosis of patients.

\section{Conclusion}

In conclusion Synchronous primary tumours in the female genital tract are rare. Adjuvant treatment is based on stage of individual primaries. The stage of presentation and extent of disease determines the prognosis of the patients.

\section{Acknowledgements}

I would like to thank Professor Dr.Sandhya Sundaram, Head of Department of Pathology for their Guidance and support and my colleagues Dr. Naveen Kumar. S and Dr. Mohan. K.P for their assistance in writing this case report.

\section{Reference}

1. Kambi DP, Mallikarjuna MN, Santosh CS et al; Synchronous malignancies of ovary, fallopian tube and cervix-A rare case. International Journal of Biomedical And Advance Research 2013; 04 [09].

2. Cheng-Kuo Lin, Mu-Hsien Yu, Ta-Wei Chul et al; Synchronus occurrence of primary neoplasms in the uterus with squamous cell carcinoma of the cervix and adenocarcinoma of the endometrium. Taiwanese Journal of Obstetrics and Gynecology. 2006;45: 336-339.

3. Hsu-Dong Sun, Chung-Ru Lai, Ming-Shyen Yen et al; Synchronous occurrence of primary neoplasms of the uterus with mucinous carcinoma of the cervix and endometrioid carcinoma of the endometrium. Taiwanese Journal of Obstetrics \& Gynecology 2011;50: 377-378

4. Seo-Yun Tong, Yong-Sek Lee, Jong-Sup Parket al; Clinical analysis of synchronous neoplasms of female reproductive tract. European Jrnl of Obs and Gyn and Reproductive Biology 2008 ; 136:78-82.

5. Eser S, Gulhan I, Özdemir R et al. Synchronous primary cancers of the female reproductive tract in Turkish Women. Asian Pacific J Cancer Prev 2011;12,857-859.

6. Axelrod JH, Fruchter R, Boyce JG. Multiple primaries among gynecologic malignancies. Gynecol Oncol 1984;18:359-72.

7. Lowery WJ, Difurio MJ, Sundborg MJ, et al. Cervical signetring cell carcinoma presenting as a synchronous primary carcinoma with uterine adenocarcinoma. Mil Med. 2009 Feb;174[2]:212-3.

8. Sakarya DK, Yetimalar MH, Demir M et al; Coexisting triple malignancy of the female genital tract with the same histopathology: An unusual case and review of the literature. Journal of Cases in Obstetrics and Gynecology, 2015;2:10-14.

*Corresponding author:

Dr Sai Shalini Chinnathambi Narayanan, Department of Pathology, Sri Ramachandra Medical College and Research Institute, Porur, Chennai, Tamil Nadu, India- 600116

Phone: +919940217743

Email: saishalini_cn14@yahoo.com

Date of Submission : 01.09.2016 Date of Acceptance : 11.02.2017

Financial or other Competing Interests: None. 